E.L.U.A., 1, 1983, págs. 227-248

\title{
PRESUPUESTOS TEÓRICOS PARA UNA GRAFÉMICA TEXTUAL
}

\author{
José Maria Jiménez Cano \\ (Universidad de Murcia)
}

Sin duda alguna, entre las aportaciones más importantes de la investigación lingüistica moderna, hay que resaltar especialmente la progresiva ampliación de las tareas y cometidos de la gramática, consecuencia, en parte, de los cambios producidos en la concepción de la misma. Si se analiza, aunque sea superficialmente este hecho, se observa que progresivamente de una concepción logicista, normativa e historicista de la gramática, basada en una consideración esencialmente representativa del lenguaje, se va pasando hacia concepciones de orientación estructural-funcional, de orientación generativotransformacional y a concepciones diversas caracterizadas por un fundamento último de carácter semiótico-comunicativo. A este último grupo mencionado pertenecen, con sus especiales características, las orientaciones de carácter textual, pragmático, psicolingüístico y sociolingüístico, que, de una parte, han agrandado el número de disciplinas configuradoras de la ciencia lingüistica, a la vez que están facilitando -ya que estamos inmersos en este proceso-, la inclusión en las gramáticas de las distintas lenguas de apartados dedicados a la exposición y explicación ordenada de estos fenómenos, cuando se adopta el reparto disciplinar en la organización del estudio de los fenómenos lingüisticos, $y$, de otra, están permitiendo la construcción de gramáticas caracterizadas por el estudio de los fenómenos lingüísticos desde la especial óptica de la nueva orientación global, caso, en particular, de 
las distintas gramáticas textuales que han llegado a proponerse:

Todavía hoy los desarrollos alcanzados por estas nuevas disciplinas no han cuajado en la elaboración de gramáticas totales o integrales de las distintas lenguas, aunque de su necesidad y presupuestos hayan hablado lingüistas como E. Coseriu y E. R. Trives (1). Por el momento la idea de integralidad es ante todo un nuevo ideal de construcción de la ciencia lingüística que podría servir de posible cierre a la situación actual descrita por L. Heilmann, para quien todavía estaríamos inmersos en el paradigma científico que inaugurara Saussure a principios de siglo (2). Resulta significativo que dentro de esta nueva orientación estén surgiendo planteamientos análogos tanto desde presupuestos estructurales-funcionales como desde presupuestos generativos. De un lado, numerosos representantes del primer grupo (especialmente $E$. Coseriu) hablan de la necesidad de proceder en la elaboración de gramáticas a la interrelación de los aspectos lingǘsticos invariables (encuadrables a nivel de sistema, de langue) y de los aspectos lingüísticos variables (localizables a nivel de discurso, de parole); de otro, los nuevos planteamientos generativos postulan la distinción entre una gramática central y una gramática periférica, como necesidad metodológica imperiosa para proceder al establecimiento de universales lingüísticos y poner las bases para un estudio contrastivo de las distintas lenguas (3). Resulta curioso comprobar que bajo estas nuevas concepciones subyacen problemas tan antiguos como el de las concepciones analógica y anómala, o el de la dialéctica constante entre unidad y variedad en el estudio de los distintos fenómenos lingüísticos, lo que contribuye a corroborar la oportunidad en la distinción de universales específicos de la ciencia lingüistica (4).

En un breve repaso histórico del reparto disciplinar de las distintas gramáticas, puede observarse cómo de un núcleo inicial casi exclusi-

(1) De E. COSERIU destaca especialmente: «Interdisciplinarità e linguaggio", en L'accostamento interdisciplinare allo studio del linguaggio, AA.VV., Milán, Franco Angeli (ed.), 1980. De E. R. TRIVES pueden tenerse en cuenta: Aspectos de semántica lingüistico-textual, Madrid, Istmo-Alcalá, 1979. Estudios sintácticos-semánticos del español (la dinámica interoracional), I, Murcia, Edt. Godoy, 1982, y «La lingüistica integral y su incidencia en el aprendizaje de una lengua extranjera", ponencia presentada al / Congreso Nacional de la Asociación Española de Lingüistica Aplicada (AESLA), Murcia, 14-17 de abril de 1983.

(2) LUIGI HEILMANN: "Del estructuralismo a la lingüistica del texto», IV Curso de Lingüistica Textual, Universidad de Murcia, abril-mayo de 1981.

(3) “ll programa chomskiano e la tipologia linguistica», de LUIGI RIZZI, en Lingua e Stile, 3,1980 , págs. $347-370$.

(4) "Los universales del lenguaje (y los otros)», de E. COSERIU, en Gramática, Semántica, Universales, Madrid, Gredos, 1978, págs. 148-205. 
vamente fonético-morfológico, con el desarrollo autónomo de las distintas disciplinas acentuado a principios de siglo, los contenidos gramaticales se van ampliando hacia lo fonológico y lo sintáctico, posteriormente hacia lo semántico (no limitado como en un principio a lo estrictamente lexicológico), para ampliarse más recientemente a problemáticas psicolingüísticas, sociolingüísticas, textuales y pragmáticas, postulándose incluso, con una cierta autonomía, ámbitos de estudio tan especificos como el de los aspectos rítmicos y entonativos de las distintas lenguas. Sin entrar para nada en las dificultades de separación y en las relaciones mutuas que mantienen los distintos niveles de análisis gramatical que se proponen, puede representarse así esta evolución:

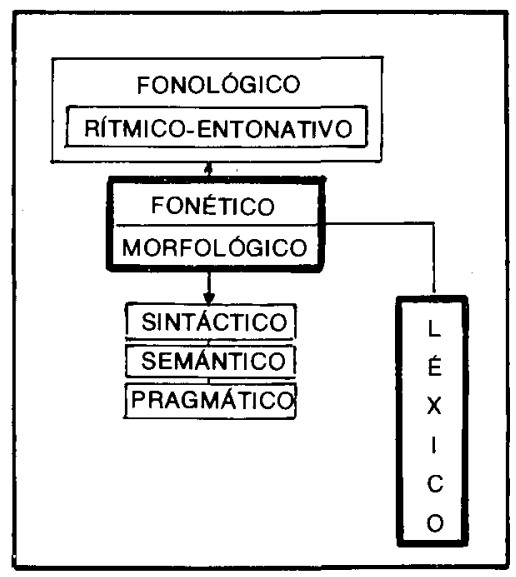

NIVELES DE

ANÁLISIS GRAMATICAL

Como detalle colateral no es ocioso recordar que el nivel pragmático tal y como aparece en el esquema es preciso entenderlo como nivel pragmático "formal» (según la caracterización de R. Montague y J.S. Petöfi (5)), ya que en 'un sentido amplio' la etiqueta «pragmático" es otra de las contendientes a alzarse con el sentido último de la orientación global de las distintas disciplinas configuradoras de la ciencia lingüistica, frente a las de "textual», "semiótico», "psicolingüístico»y "sociolingüístico", por citar tan sólo las más importantes.

A pesar de esta ampliación, uno de los aspectos que suele obviarse todavía es el de la composición o disposición gráfica y espacial, desin-

(5) “Pragmática y lógica intencional», de R. MONTAGUE, en Ensayos de filosofia formal, Madrid, Alianza, 1974, págs. 91-117. "Semantica, pragmatica, teoria del testo", de J.S. PETOFI, en La Linguistica Testuale, de M.E. Conte (ed.), Milán, Feltrinelli, 1977, págs. 195-223. 
terés que es especialmente grave en el análisis de los textos escritos y de los textos orales retorizados convencionalmente. Sin embargo, a poco que se reflexione sobre el mismo descubrimos de inmediato su importancia, en primer lugar por su presencia inexcusable en numerosos textos de lengua objeto, aquéllos que operan en el proceso comunicativo por medio de su materialidad gráfica. La composición o disposición gráfico-espacial es la conformación congénita de las realidades lingüisticas mencionadas, ya como punto final del proceso de génesis o producción lingüística, ya como punto inicial del proceso de síntesis o interpretación lingüistica - así, al menos, se manifiesta en la generalidad de los casos-. Está, además, estrechamente ligada a problemas básicos, como es el de la naturaleza lineal de los signos lingüísticos y la serie de relaciones (especialmente sintagmáticas) que les son propios. A. Martinet ofrece en este sentido un esbozo de explicación histórica:

"...le pictural tend, historiquement, à devenir graphie des langues dans la mesure où il se soumet à la linearite caracteristique du langage oral émis et perçu successivement». (6)

Pero el problema de la linealidad —como ha puesto de relieve S. Hervey- lleva aparejados numerosos problemas:

"Cette question est plus sérieuse qu'on ne pourrait le supposer, puisque la successivité de la parole qui s'écoule dans le temps est un probléme entiérement déterminé par les exigences de la "nature», alors que la successivité dans l'écriture présente des choix. Il faut avoir des conventions pour déterminer si cette successivité est d'ordre linéaire de gauche à droite, ou de droite à gauche, etc. Ceci signale une différence pertinente entre successivité dans le temps et successivité (la vrai linéarité) dans l'espace. Ce n'est donc que par usage métaphorique qu'on désigne la successivité dans le temps par le terme «linéarité». Or, on sait bien que les métaphores sont trompeuses en matiére de science.» (7)

(6) Intervención de A. Martinet en la discusión (pág. 13) de la ponencia de M. Mahmoudian: "Les rapports syncroniques de l'oral et de l'écrit au vu de l'ordre d'acquisition chez l'individu et des interdépendances ultérieures", en Actas del IV Coloquio Internacional de Lingüistica Funcional, (26-30 de julio de 1977), Universidad de Oviedo, Dpto. de Lengua Española, 1978, págs. 3-22.

(7) Intervención de S. Hervey en la misma discusión, o.c., pág. 14. 
Puede afirmarse, por tanto, que a su nivel correspondiente la disposición o composición gráfico-espacial es consecuencia última y resultante del carácter articulado del lenguaje humano.

Todo ello hace perfectamente factible el proponer como componente especialmente caracterizado, el encargado del análisis gráficocomposicional (espacial) de los textos escritos o de aquellos textos pensados para su circulación oral pero sometidos a un esquema retórico-escritural de base.

Desde un punto de vista lingüístico, el encuadre actual de estos problemas suele consistir en un capítulo especial de algunas gramáticas, dedicado a la descripción del uso convencional en una lengua de los principales signos gráficos, generalmente escriturales, con un enfoque casi exclusivamente ortográfico. Desde un punto de vista metodológico —como se expondrá más adelante—, la situación actual del estudio de estos problemas viene caracterizada por la dispersión y pluralidad de ciencias que pretenden hacerse cargo de su estudio.

Por nuestra parte, constatando la necesidad de un planteamiento interdisciplinar, proponemos el estudio de la problemática gráficocomposicional desde un punto de vista textual que permita avanzar en la elaboración de gramáticas integrales de las distintas lenguas. La adopción de esta orientación metodológica permite de antemano ajustarse a los principios básicos que orientan a un sector muy importante de la investigación lingüística moderna:

a) Una orientación global semiótico-comunicativa, con especial incidencia en los procesos de producción, recepción y traducción lingüistica.

b) La adopción de unidades de análisis superiores a la oración.

c) Mantener una actitud integradora y complementaria.

De cara a la elaboración de gramáticas de lenguas concretas este componente gráfico reagruparía aquellos elementos que de forma aislada aparecen en las mismas sin un criterio unificador y desconectado del resto de componentes. En el caso de la lengua española se trataria de analizar las numerosas aportaciones existentes (8) para intentar es-

(8) Pueden servir como ejemplos meramente indicativos y sin ningún ánimo de exhaustividad: «Ortografía», págs. 284-309, en Manual de Gramática Española, de Rafael Seco, Aguilar, Madrid, 1969. "Ortografia”, págs. 195-213, en Gramática Castellana, I Curso, de A. Alonso y H. Ureña, Losada, Buenos Aires, 1973. "Los medios de expresión», págs. 9-14, en Gramática del Español, de B. Pottier, Alcalá, Madrid, 1970. "Ortografía», págs. 58-78, en Introducción a la moderna gramática española, de J. Escarpanter, Playor, Madrid, 1974. "Ortografia", págs. 120-159, en Esbozo de una nueva gramática de la lengua española, Espasa-Calpe, Madrid, 1974. "Ortografía”, págs. 587 y sigs., en Gramática moderna del español, de M.J. Sánchez Márquez, Ediar, Buenos Aires, 1972. "El Grafema y la Ortografia”, 
tablecer una sistemática depurada, una cierta paradigmática de estos aspectos, que son aceptados de manera 'ingenua' por los hablantes y empleados con una cierta laxitud. Se trataría de localizar los límites imprescindibles que permitiesen alcanzar el objetivo de establecer la sistemática grafémico-textual de nuestra lengua. Sistemática que debería equilibrar la tendencia 'normativa', incluso 'preceptista', generalizada en los problemas de índole escritural, con el análisis de los usos específicos y el conocimiento más o menos reflexivo que los hablantes poseen de los mismos. No olvidando que precisamente en el caso de los problemas escriturales es donde con mayor fuerza se percibe la influencia institucional que la enseñanza ejerce sobre el conocimiento lingüístico de los hablantes.

La propuesta de un componente de análisis gráfico-composicional (espacial) va en la línea de potenciación del estudio de los aspectos lingüísticos materiales ('superficiales', en sentido generativo), es decir, de aquellos elementos directamente observables que han demostrado su importancia lingüística en lo relativo a los fenómenos de ordenación y configuración comunicativa del discurso, como es el caso de los fenómenos de focalización, del estudio de la dinámica tema-rema y de la dinámica de conexión y articulación de los distintos elementos que configuran el discurso humano, aspectos que en un principio no fueron valorados en su justa medida.

La especial caracterización del componente gráfico-composicional (espacial) de los distintos textos está muy estrechamente relacionada con las diversificaciones en distintos géneros y subgéneros literarios que se han producido en el transcurrir de la evolución literaria. Su estudio no se puede plantear si no es en estrecha relación con la formación histórica de los distintos géneros literarios, pues, si bien existen en todos ellos razones comunes de carácter lógico-comunicativo o estilístico en lo relativo, por ejemplo, a la puntuación y organización de los diferentes componentes textuales, cada género impone unas espe-

págs. 243-245, en Lingüistica Española, de Vidal Lamíquiz, Publicaciones de la Universidad de Sevilla, 1975. Como obra de conjunto de gran interés teorico y bibliográfico es preciso mencionar: Ortografia y ciencia del lenguaje, de José Polo, Paraninfo, Madrid, 1974. Otras obras generales que merecen destacarse son: Ortographe e Lexicographie, I, de N. Catach, J. Golfand y R. Denux, Publications du centre d'étude du français moderne et contemporain, Didier, Paris, 1971. Code Orthographique et Grammatical, de R. Thimonnier, Hatier, París, 1970. Estilistica: Teoría de la puntuación. Ciencia del estilo lógico, de Mario Linares, Paraninfo, Madrid, 1979. Para un acceso al problema desde un punto de vista histórico hay que tener en cuenta: Estudios de teoría ortográfica del español, de Abraham Esteve Serrano, Publicaciones del Dpto. de Lingüistica General y crítica literaria, Universidad de Murcia, 1982. 
ciales características gráfico-composicionales (9). Efectivamente, no son directamente equiparables la composición y distribución estrófica en el caso de la poesía versificada, con la composición de los libretos teatrales con arreglo al entretejido de actos y escenas, o la distribución por el sistema de capítulos y parágrafos como es el caso de los distintos subgéneros narrativos, en los que características como la extensión adquieren un especial valor diferenciador de las distintas especies narrativas (cuento, novela corta y novela) (10).

Esta configuración tradicional permite postular la existencia de espacios y distribuciones gráficas convencionalizadas que forman - formarian- parte de la estructura cognitivo-textual de un hablante cualificado y que inciden -incidirian-en la disposición y organización de los contenidos textuales generados. El que hablemos de hablante "cualificado" obedece al carácter selectivo de la competencia literaria desde un punto de vista socio-cultural (11).

Ejemplos de tales espacios, por tener desde el principio una referencia concreta, aunque no exhaustiva, serían, entre otros: el prólogo y la serie de textos preliminares, parágrafos, capítulos, epílogos o textos de cierre en general. Cada uno de ellos tiene razón de ser en función de un cometido especial: anticipar, dosificar o recapitular información; obedecen a unas determinadas razones de carácter comunicativo y psicológico: mantener la atención, intentar que sean memorizados de forma especial determinados contenidos. A veces, caso de los parágrafos, su especial espacialidad obedece a razones de identidad o diversidad temática o argumental (la diferente puntuación suele estar motivada por estas razones).

El hacer depender esta serie de elementos de la estructura cognitivo-textual de un hablante cualificado, supone la necesidad de conocer las convencionalizaciones cognitivas de que dispone en lo relativo a la espacialidad. Para tal estudio disponemos ya de una serie de resultados que pueden ser de utilidad para dar cuenta de este cometido:

a) J. Gérard Lapacherie ha puesto de relieve la importancia de la noción de la página como espacio y la serie de cambios históricos que han existido en la organización y disposición de lo escritural:

(9) Seria conveniente estudiar contrastivamente lo que ocurre en otras artes (pintura, escultura y arquitectura, especialmente) en lo relativo a su estructura compositiva.

(10) Cfr. M. BAQUERO GOYANES: ¿Qué es el cuento?, Columba, Buenos Aires, 1974. ¿Qué es la novela?, Columba, Buenos Aires, 1975, y Estructuras de la novela actual, Planeta, Barcelona, 1975.

(11) VICTOR M. DE AGUIAR E SILVA: Competencia lingüistica y competencia literaria (sobre la posibilidad de una poética generativa), Gredos, Madrid, 1980. TEUN A. VAN DIJK: Per una poetica generativa, II Mulino, Bolonia, 1976. 
"Les travaux sur les troubles de l'écriture (ou agraphie) ont montré, outre l'existence d'une agraphie pure, indépendante des troubles de la parole, comment l'écriture assure l'organisation de son support par un triple processus: le double alignement des mots et des lignes, si bien que la page ressemble a un rectangle noir sur fond blanc; le parallélisme entre les lignes et le respect par chacune de la linéarité de son axe. Ce triple processus codifie la mise en page et en lignes du texte: il repose sur le principe de linéarisation, qui, selon A. LeroiGourhan, est apparu à une date récente dans l'histoire de l'humanité. En effet, le graphisme scripturaire de la préhistoire se caractérise par son organisation rayonnante (alors que le notre est linéaire) et multidimensionelle. Cette double opposition (linéarité vs. rayonnance; dimensions multiples vs. dimension unique) va nous permettre d'analyser la construction de la page de 'Carte postale' ". (12).

b) En los estudios realizados sobre los conectivos, especialmente en el caso de los locativos, y sobre las manifestaciones lingüísticas de la noción de espacialidad, se acude a menudo a idealizaciones convencionalizadas del espacio que permiten entre otras cosas, la aceptación de determinadas direcciones por parte de todos los hablantes en la concepción lingüística del mismo, nociones como verticalidad, horizontalidad, disposición en diagonal. (13)

c) Igualmente en análisis crítico-literarios se ha intentado establecer los fundamentos objetivos que justifican un cambio en los modos de narrar y de describir; concretamente en el estudio de la forma de describir característica de Manzoni en I Promessi Sposi, E. Raimondi ha demostrado la incidencia de la nueva concepción científica inaugurada por Galileo en las nuevas formas narrativas que se inicían con Manzoni en el cuadro de la literatura italiana. El mismo Raimondi, al encarar el estudio de las especiales formas narrativas de Carlo Emilio Gadda, caracterizadas por la ruptura de las convenciones tradicionales en lo relativo al inicio y fin de una obra literaria, planteaba, en la misma línea de interrelación entre las especiales concepciones del mundo y

(12) “Ecriture e lecture du calligramme», pág. 197, en Poétique, 50, 1982, págs. 194-206.

(13) D. PARISI-C. CASTELFRANCHI: "Analisi semantica dei locativi speziali», en La Sintassi (Atti del III Convegno Internazionale di Studi della SLI), M. Bulzoni, Roma, 1970. CHRISTINE TANZ: Studies in the Acquisition of Deictic Terms, Cambridge University Press, 1980, P. CARBONERO CANO: Deixis espacial y temporal en el sistema linguístico, Publicaciones de la Universidad de Sevilla, 1979. 
las particulares formas narrativas, la siguiente hipótesis: las formas narrativas tradicionales no ofrecen ninguna problemática especial en lo relativo al inicio y fin narrativo de una obra literaria en la medida en que están sustentadas en concepciones cerradas del mundo no problemáticas, cuya muestra más significativa es la concepción judeo-cristiana del mundo, fundada en una visión filtrada a través de los parámetros que ofrece la Biblia, que delimita perfectamente sobre los límites iniciales (Génesis) y finales (Apocalipsis) del mundo. Otras visiones del mundo totalizantes (en lo relativo a sus limites) como la emanada de la filosofía marxista más tradicional, tampoco ofrecen dificultades de inicio o fin narrativo a aquellos escritores que se ajustan a esos presupuestos filosóficos. Típico para Raimondi de la mejor narrativa moderna y contemporánea es una visión problemática del mundo que lleva consecuentemente a desestabilizar los límites y conceptualizaciones narrativas tradicionales en los aspectos constructivos de la obra literaria. (14)

d) De especial interés, asimismo, son los estudios semióticos procedentes del establecimiento de tipologías de la cultura (Lotman, Uspenskij, Foucault) (15), y los estudios sobre el arte en general fundamentados en una psicología de la percepción (Arnheim) (16).

e) En esta fundamentación interdisciplinar que estamos efectuando, conviene, por último, acudir como soporte primario para llegar a las especificaciones de carácter cognitivo-textual, a la teoría e historia de la escritura como ejemplificación y base teórica imprescindible de las bases materiales de la producción y recepción textual. En este ámbito chocamos de antemano - ya lo anunciábamos antes-, con la dispersión metodológica de los estudios grafemáticos y escriturales, como pone de manifiesto A. Bartoli Langeli:

(14) Il romanzo senza idillio, Einaudi, Turín, 1974. Curso monográfico sobre Carlo Emilio Gadda, Bolonia, año académico 78-79. J.M. Blecua ha demostrado cómo el Quijote, fuente de toda la narrativa moderna, rompe el inicio narrativo tópico, en el análisis de los tipos de inicio textual en la narrativa anterior al Siglo de Oro (cfr. IV Curso de Lingüística Textual, Universidad de Murcia, 1981).

(15) Cfr. "Semiotica del teatro popolare", de P.G. BOGATYREV, en Ricerche Semiotiche (Nuove tendenze delle scienze umane nell'URSS), de J.M. LOTMAN y B.A. USPENSKIJ (edts.), Einaudi, Turín, 1973, págs. 5-25. all sistema spaziale nell'intreccio della bylina russam, de S. Ju. Nekljudov, en Ibídem pág. 107-124. Muy interesante como caracterización global de la cosmovisión medieval y renacentista es la ofrecida por $M$. BAJTIN en su monografía sobre Rabelais, cuando contrapone una visión vertical y jerárquica a una horizontal y progresiva (cfr. L'opera di Rabelais a la cultura popolare (Riso, carnevale e festa nella tradizione medievale e rinascimentale), Einaudi, Turín, 1979).

(16) R. ARNHEIM: Arte y percepción visual, Alianza, Madrid, 1979. GOMBRICH: Arte e illusione, studio sulla psicologia della rappresentazione pittorica, Einaudi, Turín, 1965. 
"L'atteggiamento che la Storia, professionalmente e accademicamente intesa, ha tenuto e tieni nei confronti del temascrittura è quello della parcellizzazione e della delega. Costruire tante divisioni disciplinari quanti sono, piú o meno, i tipi di prodotti scritti; affidare ciascuna a una branca di specialisti; provvedere ciascuna di una specifica attrezzatura lessicale e metodica. Ecco allora un pullulare di discipline, alcune rivendicanti una piena autonomia (storia della letteratura, storia della lingua scritta/parlata, filologie varie), altre tradizionalmente ascritte al vario sistema delle cosiddete scienze ausiliari della storia e, spesso, alla laboriosa ricerca dell'emancipazione: paleografia, diplomatica, codicologia, bibliografia, storia del libro, storia della tradizione manoscrita...»(17).

Tal dispersión cabría explicarla, en opinión de Bartoli, por la amplitud de fuentes materiales y la serie de problemas teóricos que llevan aparejadas:

«...assumere come fonti tutti i materiali che attengono, direttamente e indirettamente, alla scrittura. Quindi, il libro ma non solo il libro: appunti, documenti, sottoscrizioni, scrittura d'apparato, scritture murali e cosí via. Ciascuna delle evidenze materiali della scrittura rimanda a un processo culturale, a un tipo di alfabetismo, a una forma di socialitá, e pertanto merita un'analisi, una descrizione e una formalizzazione in quanto. tale." (18).

Para este mismo autor la amplitud e importancia de los materiales escriturales tiene una incidencia inmediata en la organización cultural de una sociedad:

«Si puó tuttavia aggiungere un terzo tema "globale» di studio...: le procedure e istituzioni attraverso le quali ogni sistema sociale provvede ad alfabetizzare e a far circolare la cultura scritta: la scuola certamente, il libro e il sistema editoriale certamente, ma anche i mezzi di comunicazione e persuasione "pubblica", la scrittura come regolatore ordinario della vita collettiva, la lingua standard, le strutture del discorso...» (19).

(17) “Premessa» de A. Bartoli (pág. 438) a “Alfabetismo e cultura scritta», en Quaderni Storici, 38, Ancona, mayo/agosto de 1978.

(18) Ibidem, pág. 441.

(19) Ibidem, pág. 445. 
Incidencia en la organización cultural que le lleva a plantearse hasta qué punto lo escritural no impone una determinada manera de ver el mundo:

«E proprio la natura istituzionale e statutaria della scrittura, la quale consente di radicare $\mathrm{i}$ fenomeni culturali nel vivo della struttura sociale, di ancorare le «idee" ai meccanismi oggettivi della produzione e propagazione culturale" (20).

Las tareas propuestas por estas direcciones de estudio son perfectamente asumibles desde un punto de vista textual. A. Petrucci enumera las siguientes:

«1) Il meccanismo dell'insegnamento del leggere e dello scrivere nelie sue diverse articolazioni sincroniche (sociali e geografiche) e diacroniche; 2 ) i modi di realizzazione e le caratteristiche dell'uso passivo della cultura scritta, e cioè della lettura, sia di libri che di ogni altra testimonianza grafica; ed anche della ricezione visuale del messaggio puramente esteticoformale che pure ogni scrittura (soprattutto se di apparato) contiene e trasmette; 3 ) la differente dislocazione del grado di alfabetizzazione (attiva e passiva) nei vari settori di una società divisa in classi; 4) le scelte ideologiche ed economiche che di volta in volta sono alla base del processo di produzione (manoscrito o a stampa) dei prodotti grafici (e in particolare del libro), i meccanismi e gli strumenti tecnici per mezzo dei quali nelle varie epoche esso si esplica, e il peso che in esso esercitano i protagonisti umani, che comunque vi partecipano a diversi livelli e con differenti responsabilitá». (21)

No va a ser cometido del presente estudio plantear en toda su extensión la problemática de la relación entre lo fónico y lo gráfico. Baste con exponer las actitudes dominantes que podrian quedar resumidas en las siguientes tendencias:

a) Tendencia a la indistinción entre ambos: la substancia, desde este punto de vista (22), puede ser o 'fónica' o 'gráfica', si la substancia

(20) Ibídem.

(21) "Per la storia dell'alfabetismo e della cultura scritta: metodi, materiali, quesiti", pág. 452, en aAlfabetismo e cultura scritta», O.c., págs. 451-465.

(22) M. A. K. HALLIDAY: "Le categorie della teoria della grammatica", pág. 114, en La linguistica: aspetti e problemi, de L. Heilmann-E. Rigotti (edts.), II Mulino, Bolonia, 1975, págs. 111-151. 
es fónica es reconducida a la forma por la fonología; si la substancia es gráfica es reconducida a la forma por la ortografía o grafologia. Como se observa la única distinción es de carácter metodológico pero no se indaga teóricamente la base de esa posible diferencia.

b) Tendencia a establecer la prioridad de lo fónico y consecuentemente afirmar el carácter derivado de lo gráfico:

«Nous refusons l'interprétation de certaines théories linguistiques qui font abstraction de la substance meme dans laquelle se réalise la langue, et considerent la représentation graphique, aussi bien que l'expression phonique, comme deux manières possibles de matérialiser le systeme formel unique qu'est la langue. Pour nous, le langage a une manifestation normale et primaire, qui est phonique; l'écriture, ou représentation graphique, étant sa manifestation sécondaire: du point de vue linguistique, on ne peut l'étudier en elle-même, mais seulement dans ses relations avec la première». (23)

Tanto una como otra ha llevado a afirmar a determinados autores la existencia de un fonocentrismo en el pensamiento lingüístico occidental (24).

Como visión compendiadora de la relación fónico-gráfica puede servir la ofrecida por L. Heilmann, fundada en el siguiente esquema:

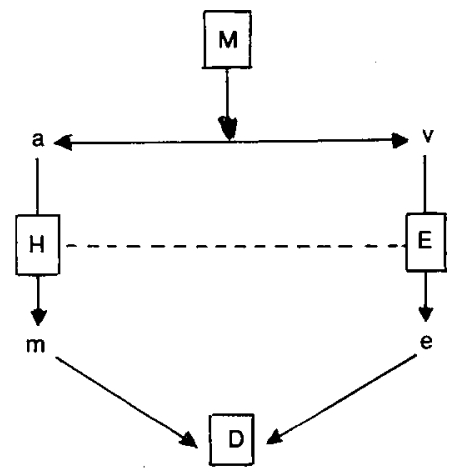

(23) E. ALARCOS LLORACH: "Les représentations graphiques du langagem, págs. 519520, en Le langage, de A. Martinet (ed.), Gailimard, París, 1968, pág. 515-568. En este mismo sentido: M. ALVAR: “Fonética, Fonología y Ortografía», en Lingüistica Española Actual, 1/2, 1979, págs. 211-231.

(24) "Le fonocentrisme de la pensée occidental définit le langage par l'oralité et ramène le signe et ses signifiants à una séquence sonore. Le signe étant «une suite de sons se succédant 
Esquema que ha de entenderse del siguiente modo: en la transmisión de un mensaje (M) a un destinatario (D), la escritura (E) constituye la traducción de la lengua hablada $(\mathrm{H})$ insertándola en el eje visualestable ( $v$-e) en forma paralela al eje acústico momentáneo $(\mathrm{a}-\mathrm{m})$ en el que se inserta la expresión oral. La escritura sería una comunicación visual estable que traduce por medio de signos convencionales (grafemas) el mensaje oral, permitiendo su conservación en el tiempo y su transmisión en el espacio (25).

La operatividad del componente gráfico-composicional no se agota en su carácter de traducción visual y estable de lo fónico, sino que, además, debe dar cuenta, como ya se ha expuesto, del conocimiento y del uso por parte del hablante de los elementos compositivos y organizadores que garantizan la construcción y manifestación externa de los textos escritos. El siguiente esquema puede clarificar ambos aspectos:

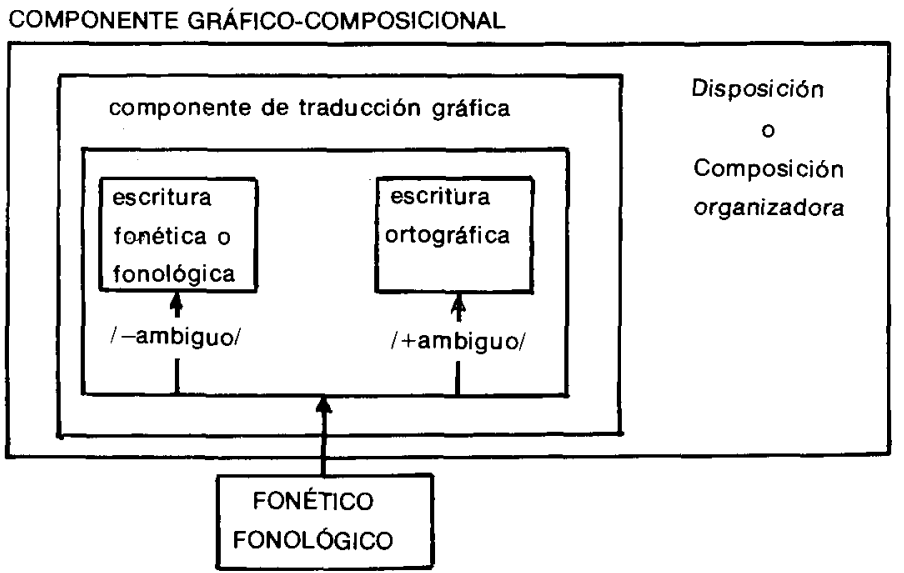

das le temps», G. E. Lessing exclut du discours littéraire la représentation de l'espace et occulte la dimension graphique, spatiale, visuelle de tout texte inscrit sur un support. Du phonocentrisme qui imprègne la linguistique du XXe siècle procède la mèconnaissance de l'écriture, conçue comme un "signifiant de signifiant». Le premier signifiant es phonique; le second, graphique. Pour J. Derrida, au contraire, l'écriture est atrace», a partir de laquelle un sens peut etre etabli. A. Leroi-Gourhan pose l'existance d'un «langage visuel", dont le prototype est l'écriture et qui permet à l'homme d'exprimer une pensée par des images, des figures, des formes inscrites. L'autonomie de l'écriture par rapport a la parole est aussi une these du linguiste tcheque J. Vachek, qui oppose un *langage écrit w au langage oral, et de . Hjelsmlev, pour qui la forme linguistique, algébre de relations, peut s'actualiser dans n'importe quelle substance, iconique, phonétique, graphique, gestuelle." J. G. LAPACHERIE: "Ecriture et lecture du calligramme», art. cit. págs. 194-195.

(25) L. HEILMANN: «Introduzione», pág. 14, en La linguistica: aspetti e problemi, o.c., págs. 13-34. 
La disposición o composición organizadora coincide con lo que Greimas, apoyado en criterios pragmáticos espacio-temporales, denomina dispositif graphique (26).

Desde el segundo punto de vista, el principal problema por solucionar es establecer y clarificar las relaciones que el componente gráficocomposicional establece con el resto de componentes que intervienen en la creación de un texto. Tomando como referencia el proceso de génesis textual pueden ser establecidas dos orientaciones diferentes:

a) Una orientación convencionalizada en la que el componente gráfico-composicional es el ajuste final organizador del resto de componentes. En estos casos la disposición final no es problemática y se ajusta a las convenciones constructivas del particular tipo de texto que se trate de confeccionar. El usuario lingüístico dispone desde un principio del marco gráfico-composicional al que ajusta los resultados de los demás componentes con arreglo a la disposición o reglamentación convencionalizada.

b) Una orientación no convencionalizada en la que el resto de componentes, sin perder su operatividad propia, se subordinan en cierta medida al componente gráfico que adquiere en consecuencia, una especial importancia. Sería éste el caso de los caligramas, de los distintos juegos anagramáticos y de los juegos con el orden variable de lectura de los distintos componentes de un texto literario. En este sentido ha llegado a hablarse de la escritura como gesto (27). En el caso de los juegos anagramáticos es conveniente advertir que cuando proceden de textos pensados para su circulación oral, hay que considerarlos como de intencionalidad fónica; sólo el cambio sociocultural impuesto por la circulación de los textos en forma impresa motiva la prioridad de

(26) “Le texte choisi, sous sa forme écrite, comporte un dispositif graphique caractérisé par le choix des caractères d'imprimerie, le decoupage phrastique, le découpage en paragraphes, etc., tout discours... présente une organisation multiplane..., sa mise en paragraphe peut correspondre à des délimitations indiscutables, mais situées tantôt sur l'un tantôt sur l'autre des niveaux du déroulement discursif. Aussi est-on amené le plus souvent à recourir, en primer lieu, aux critères spatio-temporels de segmentation, qui ont l'avantage d'etre uniformément présents dans tout discours pragmatique, c'est-à-dire dans le discours relatant des séries «d'événements" ou de "faits" qui, eux, se trouvent nécessairement inscrits dans le système de coordonnées spatio-temporelles». Citado por E.R. Trives en Aspectos de Semántica lingüístico-textual, o.c., pág. 224, nota 498.

(27) Pueden ser tenidos en cuenta los siguientes trabajos: el ya mencionado de J.G. LAPACHERIE, “Los anagramas de Ferdinand de Saussure (textos inéditos) » de J. Starobinski, en $F$. de Saussure: Fuentes manuscritas y estudios criticos, de Ana María Nethol (ed.). Siglo XXI, Méjico, 1977, págs. 229-247, y «La lettre et les idéogrammes occidentauX», de J.C. COQUET, en Poétique, 11; 1972, págs. 395-404. 
lo gráfico. También habría que incluir aquí el caso de la creación léxica a partir de errores gráficos (28).

Esta doble orientación en el componente gráfico-composicional puede ser utilizada como uno más entre los criterios para el establecimiento de una tipología de los distintos textos.

Salvo en el caso de la segunda orientación en la que el componente gráfico-composicional adquiere un cierto carácter entitativo, su característica esencial es la dependencia o subsidiariedad con relación al resto de componentes. En concreto, W. Dressler en las dos páginas que dedica en su obra de introducción a la lingüística del texto a la grafémica y a los textos escritos, considera que esta problemática sólo alcanzará una explicación cuando se disponga de un conocimiento más preciso del resto de componentes, especialmente el componente de base temático-semántico:

«Solo quando sará possibile predire piú essattarnente la derivazione della struttura superficiale sintattica del testo a partire da un componente di base di un testo tematico e semantico del testo, potremo piú chiaramente comprendere i mutamenti avvenuti nel passaggio alla fonologia del testo e stabilire con piú precisione quando questa debba esser fatta derivare direttamente dalla semantica del testo.» (29)

Insistiendo en esta dependencia semántica, advierte asimismo de la dificultad de proceder a generalizaciones en el estudio de los elementos gráfico-textuales:

«Ogni specie di interruzione dello sviluppo semantico è un in-

(28) Reproducimos, dado su interés, la amplia cita de Lotman:

«Analizzando i manoscritti di Puśkin ci si convince che in certi casi ci sono tracce dell'influenza sullo svolgimento successivo dell'opera di errori evidenti, che suggeriscono la rima successiva e incidono sullo sviluppo della narrazione. Cosi, analizzando la brutta copia della poesía «Vcě ticho, na Kavkaz idét nočnaja mgla» (Tutto e tranquillo, sul Caucaso si stende la caligine notturna) S. M. Bondi in un solo manoscrito ha trovato due di questi casi: 1) «La lettera «e», della parola «legla» (si stendeva) è stata scritta da Puskin senza l'occhieIlo, cosicché la sua grafia veniva a coincidere per caso con la grafia della parola mgla (caligine). Questo errore casuale della penna non ha forse indicato al poeta la via verso la variante "idët nočnaja mgla» (si stende la caligine notturna)?»...

2) "La parola net (no) e stata scritta da Puškin in modo tale che poteva passare per let (anni). Transformando cosl mnogich net (molti no) in mnogich let (molti anni)... Gli esempi riportati confermano che le alterazioni meccaniche in certi casi possono apparire come riserva della riserva (riserva della fascia extrasistematica del testo)n. Testo e contesto (Semiotica dell'arte a della cultura), Laterza, Bari, 1980, pág. 21.

(29) W. DRESSLER: Introduzione alla linguistica del testo, Officina, Roma, 1974, pág. 118. 
dizio per la delimitazione delle parti del testo, o di gruppi di frasi o di stringhe, paragrafi, capitoli, ecc., appartenenti alio stesso insieme. La presenza dei mutamenti rende naturalmente piú difficile il preciso riconoscimento degli esatti confini dei segmenti di testo. Inoltre la costituzione dei segmenti di testo, per esempio dei paragrafi, è molto diversa a seconda della lingua, cultura, stile e genere del testo, ed è quindi difficile generalizzare. Decisiva è la relativamente maggiore coerenza semantica tra frasi di uno stesso frammento di testo considerato. Questa coerenza dovrebbe fondarsi più sul rapporto con il tema generale del segmento che sulla contiguità semantica delle singole frasi. Ancora una volta, quindi, l'omogeneità non è in superficie.» (30)

El plantearse o no lo gráfico como algo autónomo, desligado de los procesos que instauran los demás componentes, dependerá del tipo de texto o de género que en cada caso se considere. Argumento para la posible autonomía del componente gráfico-composicional sería el hecho de disponer él mismo de una articulación por niveles. En efecto, podría hablarse de una especie de sintaxis -en cuanto articulación de una serie de unidades o componentes—, de una cierta semántica, en la medida en que el juego con las disposiciones convencionales puede

(30) Ibídem, pág. 96. Otra visión de lo gráfico-composicional como dependiente o integrada es la ofrecida por $M$. Bajtin que resumimos con algunas de sus afirmaciones:

“...les mots, les phonèmes, les morphemes les propositions et les séries sémantiques: ceux-ci sont placés hors du contenu de la perception esthétique, c'est-a-dire hors de l'objet artistique; ils ne peuvent servir qu'à un jugement scientifique au second degré de l'esthétiquex. (Pág. 62). «Dans une oeuvre poétique, les mots s'organisent d'une part selon les ensembles que sont les propositions, les périodes, les chapitres, les actes, etc., d'autre part, ils construisent l'aspect du personnage, son caractère, sa situation, son cadre, ses actes, et enfin l'ensemble que forme l'événement éthique d'une vie, mis en forme et parachevé de façon esthétique; ce faisant, ils cessent d'etre mots, des propositions, des lignes, des chapitres: le processus de réalisation de l'objet esthétique, autrement dit, du dessein artistique dans son essence, est un processus de transformation systématique, d'un ensemble verbal, compris linguistiquement et compositionnellement, en l'ensemble architectonique d'un événement esthétiquement achevé; bien entendu, toutes les liasons et interactions verbales d'ordre linguistique et compositionnel se transforment alors en liaisons architectoniques, extra-verbales* (pág. 63).

“ll faut souligner le caractere auxiliare de l'organisation matérielle de l'oeuvre, son caracter proprement technique, non pour la rabaisser, mais au contraire pour lui donner un sens et la vivifier (68).

“...dans le roman, et en général dans les plus grands ensembles verbaux en prose, le phonème cède presque complètement ses fontions auxiliares (désigner le sens, provoquer un mouvement, servir de base à l'intonation) au graphème. (Pág. 78). En "Le probleme du contenu, du matériau et de la forme dans l'oeuvre littéraire», en Esthétique et théorie du roman, págs. 23-82. 
resultar significativo, y, por último, de una cierta pragmática cuando, por ejemplo, determinados ingredientes de espacio y composición pueden 'presentar' un texto como poema, como diálogo teatral, etc., o dotarlo de unas determinadas atribuciones. Ejemplo privilegiado de esta posible autonomía del componente gráfico-composicional seria su especial importancia en los medios escritos de comunicación de masas (especialmente en la prensa). También dentro de lo pragmático juegan un importante papel las connotaciones sociológicas, ya que, por ejemplo, el aparato composicional puede servir para diferenciar al libro culto del de circulación popular (especialmente en otros momentos históricos) (31). De igual manera puede ser considerado como criterio para su posible autonomía, la comprobación desde un punto de vista contrastivo del componente gráfico como primer elemento de conocimiento en el acceso a un texto escrito, en base a la convencionalidad más o menos generalizada de la disposición gráfica. Por último, se pueden aducir razones históricas en la manera de ser adquiridos los conocimientos gráfico-composicionales (la escritura como opus servile) (32).

La importancia del papel del componente gráfico-composicional viene reforzada por el hecho de la existencia histórica de la que puede denominarse noción de texto como idea (ideación) de libro o de obra literaria. Esta equiparación entre texto y obra literaria se producía en la

(31) «Si consideri la suddivisione interna di questi libretti in tanti blocchi regolari, indifferenziati al loro interno, con uso ridottissimo de techniche esplicitanti (capitoli, paragrafi, sottoparagrafi, capoversi, di nuovo punteggiatura): Blocchi, unità di lettura corrispondenti forse a una misura standard di templo di lettura. Lo stesso procedere del discorso o della narrazione, che "avanza lentamente, con frequenti riprese, ripetizioni i precisazioni", sembra configurare un modo, funzionale alla lettura semialfabetica, di apprendere e isolare $i$ punti nodali dello sviluppo testuale, di «seguire il filo del discorso". A tali scopi invence il libro colto dedicava indici, numerazionim titolazioni articolate e gerarchiche, sussidi visuali. Accorgimenti che facilitavano anche il atornare in dietrom, il ritrovare un passo precedente: operazioni importanti per "capire» e, come ben si comprende, a tal punto ardue da risultare impraticabili per un lettore semialfabeta. Sia il tipo di lettura sia la struttura del libro che ne rispecchiava e accentuava il procedimento, erano chiaramente inidonei a dare al lettore - se, infine, puo chiamarsi lettoro- un senso coerente e complessivo del testo letto. Per rendere l'idea, paragonerei questo modo di lettura a quello degli studenti che affrontano generosamente i primi rudimenti della paleografia: totalmente impegnati a superare l'ostacolo frapposto dagli elementi sia grafici, sia linguistici, sia «strutturali» (lontani tutti in varia misura dai propri sistemi usuali), essi comprendono ben poco, ahimé, di quanto leggonom. En «Premessa de A. Bartoli, cit. págs. 448-449.

(32) «Diventare un esperto di qualsiasi tipo grafico costava, dunque, un lungo e faticoso apprendistato; ma l'artigiano, in generale, non aveva alcuna intelligenza dei contenuti, non capiva quanto scriveva, si contentava di applicare servilmente le tecniche che gli erano state insegnate nel corso del suo tirocinio. La scrittura era e restava, almeno fino all'età diocleziana, opus servilem. En «Dal segno incompiuto al segno negaton, pág. 480, en «Alfabetismo e cultura scritta», o.c., págs. 466-487. 
tradición filológica y se prolonga, incluso, en las visiones estructurales y narratológicas. Como señala W. Dresler:

«ll concetto di testo...non compare nelle grammatiche scolastiche se non nel significato di libro 0 , ancora più in generale, di qualcosa scritta in parole." (33)

La imagen de texto como obra literaria es patente en la orientación estructural:

«Frente a un texto... todo deberá ser considerado minuciosamente. Desde el título hasta el punto final. Todo puede ser indicio que ponga de manifiesto ese orden superior que gobierna las relaciones internas y del cual nace el sentido y la organización estructural.»(34)

Y en las posiciones narratológicas subyace al fondo la misma idea:

"La noción de texto no se sitúa en el mismo plano que la de frase (o la proposición, el sintagma, etc.); en este sentido, el texto debe distinguirse del parágrafo, unidad tipográfica de varias frases." (35)

Serán las orientaciones textuales de base metodológica hipotéticodeductiva las que intenten ir más allá de esta visión, a la que califican de 'ética' (noción de texto como unidad del discurso, de la paro/e):

"Quelle definizioni del testo che prendono in considerazione solamente i testi etici sono insufficenti.» (36)

De ahí que propongan una definición desde un punto de vista 'émico' (como unidad del sistema, de la langue):

«invece de tali vaghi tentativi di definizione, un testo emico dovrebbe servire piuttosto come punto indefinito di partenza del processo linguistico, cioè come simbolo $T$ di una rappre-

(33) W. DRESSLER, o.c., pág. 9. 21.

(34) R. H. CASTAGNINO: 'Sentido' y estructura narrativa, Nova, Buenos Aires, 1975, pág.

(35) DUCROT-TODOROV: Diccionario enciclopédico de las ciencias del lenguaje, Siglo XXI, Argentina, 1974, pág. 337.

(36) W. DRESSLER, o.c., pág. 24. 
sentazione generativa, del quale deriva un componente semantico di base conchiuso che da parte sua è l'entrata dell'insieme di regole della sintassi del testo e della frase.» (37)

Se inaugura de esta forma una contradicción entre una definición realista, ética, inductiva del texto (Coseriu, principalmente (38)) y una definición formal, émica, hipotético-deductiva (Van Dijk, Petöfi, principalmente (39)), que conciben el texto, respectivamente, como unidad de la parole, del discurso, o como unidad del sistema de la langue y, por tanto, susceptible de una definición abstracta.

La noción de texto como idea (ideación) de libro está basada en la imagen variable históricamente de las caracteríticas estereotipadas de la idea de libro u obra literaria (en sus distintos géneros) en lo relativo a su carácter, construcción y función. Como recuerda J. M. Lotman:

«Nel corso dell'evoluzione storica è venuto un momento in cui il numero dei testi soggetti all'azione della memoria ha superato la possibilitá della memoria individuale. Ė sorta allora una cultura scritta che ha dato la posibilità di fissare nella memoria della collettività un numero illimitato di testi. Il significato della memoria scritta è stato tale che le immagini di un libro o di una biblioteca hanno cominciato ad identificarsi nella coscienza degli uomini con lo stesso concetto di memoria.» (40)

Podriamos recordar como ejemplo de imagen global (41) la ofrecida por el Arcipreste de Hita en su Libro de Buen Amor como instrumento musical. Como ejemplo privilegiado de la imagen convencionalizada del carácter, construcción y función de un tipo de texto concreto se puede aducir la que aparece en clave irónica en el prólogo de Cervantes a la primera parte del Quijote.

Consecuencia teórica de esta noción de texto como idea (ideación) de libro es la de permitir replantear la noción de competencia literaria (especialización necesaria de la noción de competencia lingüística en ámbito literario) como conciencia metalingüístico-textual, es decir, como capacidad de producir e interpretar textos con arreglo a una

(37) Ibídem, pág. 25.

(38) E. COSERIU: Textlinguistic: eine Einfärung, Gunter Narr, Tübingen, 1981.

(39) A. GARCIA BERRIO-T. ALBALADEJO MAYORDOMO: "La lingüistica del texto", en Introducción a la lingüistica. AA.VV., Alhambra, Madrid, 1982, págs. 217-260.

(40) Testo e contesto, o.c. pég. 43.

(41) ANTONIO PRIETO: Coherencia y relevancia textual (De Berceo a Baroja), Alhambra, Madrid, 1980. 
convencionalización relativamente general de los mismos que permite proceder a la producción o recepción textual bien desde una orientación analógica, ajustando las caracteristicas del texto por producir o interpretar a las generalmente aceptadas, bien desde una orientación innovadora o desautomatizadora variando en determinados aspectos esas características generales. Desde un punto de vista textual la función metalingüística propuesta por $\mathrm{R}$. Jakobson pasaría a ocupar una funcionalidad al mismo tiempo previa y globalizadora.

Se hace, pues, necesario identificar los distintos elementos que intervienen en esa convencionalización relativamente general que sirve de base a la conciencia metalingüístico-textual. Con el presente trabajo queremos defender la idea de que precisamente el que hemos denominado como componente gráfico-composicional es uno de los que en mayor medida contribuyen a esa convencionalización.

Especial incidencia posee dentro del amplio dominio de la composición textual la que puede denominarse como topología textual, es decir, la articulación o sintagmática de los distintos componentes de un texto. En efecto, concebida la composición como distribución espacial, puede afirmarse que todo texto se configura en este sentido en torno a un núcleo o eje central al que se refieren de un modo directo o indirecto una serie de subtextos que pueden ser etiquetados como satélites. La disposición espacial de los textos satélites forma parte de su propia naturaleza textual y en razón de ella actúan como los límites demarcadores del texto en su conjunto, ya sea indicando su inicio, su transcurrir, su cierre o conclusión. Textos satélites indicadores del inicio pueden considerarse el título, el prólogo y sus variantes (advertencias, prefacios, introducciones, preámbulos, etc.), la dedicatoria y las citas previas; indicadores del transcurrir pueden considerarse la repetición especificada de los elementos de inicio o el entretejido de citas y de referencias a otros contextos textuales; por último pueden ser considerados como indicadores de cierre los distintos tipos de epilogo y sus variantes. La distribución topológica convencionalizada viene justificada por razones psicológicas y de lógica comunicativa (satisfacer la espera de información, la aclaración progresiva, la ratificación final, por ejemplo), las mismas en las que, por otra parte, se asentaba la disposición del discurso oratorio que, en gran medida, sigue siendo un factor condicionante (téngase en cuenta, por ejemplo, la función retórica exordio y su relación con los textos satélites indicadores del inicio textual).

El ajustarse a un mayor o menor grado de convencionalidad en la disposición de los textos escritos puede interpretarse como el deseo por parte del autor del texto de implicar al lector en mayor o menor 
medida. Desde este punto de vista han de explicarse los efectos narrativos conducentes a la creación de suspense, la confección de prólogos apócrifos con finalidad organizadora del texto que presentan (el prólogo de Los usurpadores de F. Ayala) o la creación de finales narrativos múltiples (El astillero, de J.C. Onetti). De todas formas el más claro ejemplo del uso metalingüístico del espacio textual es el de la serie de referencias convencionalizadas de carácter anafórico y catafórico en la dinámica intratextual o cotextual.

Hasta ahora todas las consideraciones que hemos efectuado del componente gráfico-composicional se encuadran dentro de una dimensión de estudio sincrónica; sin embargo, como el resto de aspectos lingüísticos la problemática gráfica y composicional es susceptible de ser estudiada diacrónicamente. El estudio diacrónico del componente gráfico-composicional puede ser planteado en dos dimensiones de estudio complementarias:

a) Una dimensión extrínseca apoyada en un estudio interdisciplinar con materias tales como la historia del libro o la sociología de la lectura, que daría cuenta de la configuración histórica de la imagen de texto como ideación de libro. Es el criterio adoptado por F. Rigolot, quien frente al dilema de abandonar la noción de texto por su carácter polisémico o por ser susceptible de definición sólo negativamente, prefiere acudir al estudio de la lenta elaboración filológica de esta noción, deteniéndose de forma especial en sus momentos clave: las retóricas antiguas (Aristóteles y Quintiliano) y el humanismo filológico del Renacimiento (42).

b) Una dimensión intrínseca que daría cuenta de los cambios lingüisticos textuales producidos en la disposición y organización gráfica e intentaría dar cuenta de los mismos (cambio textual en la disposición textual de la dedicatoria que pasa de estar construida en forma de carta al simple sintagma preposicional).

Un posterior uso aplicativo de los presupuestos teóricos para una grafémica textual que acabamos de exponer, requiere la especificación clara de los distintos niveles del estudio lingüístico propuesta por $\mathrm{E}$. Coseriu, agrupando los fenómenos gráfico-composicionales según su operatividad, ya sea ésta universal, idiomática o discursiva.

(42) «La renaissance du texte (histoire et sémiologie)», en Poétique, 50, 1982 págs. 183-193. 